\title{
Boundary Values of Generalized Harmonic Functions Associated with the Rank-One Dunkl Operator
}

\author{
Jiaxi Jiu and Zhongkai $\mathrm{Li}^{*}$
}

Department of Mathematics, Shanghai Normal University, Shanghai 200234, China

Received 2 January 2020; Accepted (in revised version) 12 January 2020

Dedicated to Professor Weiyi Su on the occasion of her 80th birthday

\begin{abstract}
We consider the local boundary values of generalized harmonic functions associated with the rank-one Dunkl operator $D$ in the upper half-plane $\mathbb{R}_{+}^{2}=\mathbb{R} \times$ $(0, \infty)$, where

$$
(D f)(x)=f^{\prime}(x)+(\lambda / x)[f(x)-f(-x)]
$$

for given $\lambda \geq 0$. A $C^{2}$ function $u$ in $\mathbb{R}_{+}^{2}$ is said to be $\lambda$-harmonic if $\left(D_{x}^{2}+\partial_{y}^{2}\right) u=0$. For a $\lambda$-harmonic function $u$ in $\mathbb{R}_{+}^{2}$ and for a subset $E$ of $\partial \mathbb{R}_{+}^{2}=\mathbb{R}$ symmetric about $y$-axis, we prove that the following three assertions are equivalent: (i) $u$ has a finite non-tangential limit at $(x, 0)$ for a.e. $x \in E$; (ii) $u$ is non-tangentially bounded for a.e. $x \in E$; (iii) $(S u)(x)<\infty$ for a.e. $x \in E$, where $S$ is a Lusin-type area integral associated with the Dunkl operator $D$.
\end{abstract}

Key Words: Dunkl operator, Dunkl transform, harmonic function, non-tangential limit, area integral.

AMS Subject Classifications: 42B20, 42B25, 42A38, 35G10

\section{Introduction and main results}

For given $\lambda>0$, the rank-one Dunkl operator on the line $\mathbb{R}$ is defined by

$$
(D f)(x)=f^{\prime}(x)+\frac{\lambda}{x}(f(x)-f(-x)) \text {. }
$$

A $C^{2}$ function $u$ in the upper half-plane $\mathbb{R}_{+}^{2}=\mathbb{R} \times(0, \infty)$ is said to be $\lambda$-harmonic if $\Delta_{\lambda} u=0$, where

$$
\Delta_{\lambda}=D_{x}^{2}+\partial_{y}^{2}
$$

${ }^{*}$ Corresponding author. Email addresses: jiujiaxi78@163.com (J.-X. Jiu), lizk@shnu.edu.cn (Z.-K. Li) 
The operator $\Delta_{\lambda}$ is called the $\lambda$-Laplacian, and can be written explicitly by

$$
\left(\Delta_{\lambda} u\right)(x, y)=\frac{\partial^{2} u}{\partial x^{2}}+\frac{\partial^{2} u}{\partial y^{2}}+\frac{2 \lambda}{x} \frac{\partial u}{\partial x}-\frac{\lambda}{x^{2}}(u(x, y)-u(-x, y))
$$

Some aspects of harmonic analysis in the upper half-plane $\mathbb{R}_{+}^{2}$ associated to the Dunkl operator $D$ were studied in [25] and their analogues in the unit disk $\mathbb{D}$, associated with Dunkl-Gegenbauer expansions, were developed in [26]. These are generalizations of the seminal work of Muckenhoupt and Stein [31] on the Bessel operator and the Gegenbauer expansions. In this paper we study the local existence of boundary values of $\lambda$-harmonic functions in the upper half-plane $\mathbb{R}_{+}^{2}$.

It is well known that, if $u$ is a harmonic function in the unit disk $\mathbb{D}$ and $E$ is a subset of positive measure of the boundary $\partial \mathbb{D}$, then the existence of non-tangential limit at almost every $e^{i \theta} \in E$ of $u$ can be characterized by non-tangential boundedness of $u$ at almost every $e^{i \theta} \in E$, and also by finiteness of Lusin's area integral of $u$ at almost every $e^{i \theta} \in E$. The former, as a local version of Fatou's theorem, was owed to Privalov [38], and the latter was proved by Marcinkiewicz and Zygmund [30] and Spencer [42]. One of the basic tools in these works is the conformal mapping, which introduces technical difficulties in extending them to more variables and other settings. Calderón $[5,6]$ made a breakthrough and generalized Privalov's theorem and Marcinkiewicz and Zygmund's theorem to Euclidean half-spaces of several variables by the real-variable method. A generalization of the theorem of Spencer [42] to several variables was obtained in Stein [43]. Since then, criteria on existence of non-tangential boundary limits of harmonic functions in many different contexts, in terms of non-tangential boundedness or one-side non-tangential boundedness or finiteness of area integrals have been intensively studied; see, for example, $[1-4,7,14-22,24,32-37,39]$ and $[46]$.

As usual, we denote by $\Gamma_{\alpha}(x)$ the positive cone of aperture $\alpha>0$ with vertex $(x, 0) \in$ $\partial \mathbb{R}_{+}^{2}=\mathbb{R}$, and $\Gamma_{\alpha}^{h}(x)$ the truncated one with height $h>0$, that is,

$$
\Gamma_{\alpha}^{h}\left(x_{0}\right)=\left\{(x, y) \in \mathbb{R}_{+}^{2}:\left|x-x_{0}\right|<\alpha y, 0<y<h\right\} .
$$

For a function $u$ defined in $\mathbb{R}_{+}^{2}$ and for $\alpha>0$, the non-tangential maximal function $u_{\nabla}^{*}(x)$ is defined by

$$
u_{\nabla}^{*}(x)=\sup _{(t, y) \in \Gamma_{\alpha}(x)}|u(t, y)|
$$

that $u$ has a non-tangential limit at $(x, 0)$ means that for every $\alpha>0, \lim u(t, y)$ exists as $(t, y) \in \Gamma_{\alpha}(x)$ approaching to $(x, 0)$; and that $u$ is said to be non-tangentially bounded at $(x, 0)$ if $u(t, y)$ is bounded in $\Gamma_{\alpha}^{h}(x)$ for some $\alpha, h>0$. For a $C^{2}$ function $u$ in $\mathbb{R}_{+}^{2}$, we define the Lusin-type area integral $S u=S_{\alpha, h} u$ for some $\alpha, h>0$ by

$$
\left(S_{\alpha, h} u\right)(x)=\left(\int_{\Gamma_{\alpha}^{h}(0)} \tau_{x}\left(\Delta_{\lambda} u^{2}\right)(-t, y) y^{-2 \lambda}|t|^{2 \lambda} d t d y\right)^{1 / 2}
$$


where $\tau_{x}$, acting on the first argument, is the associated (generalized) translation in the Dunkl setting (see Section 2). We note that $S u$ was first defined in [27] (see [28] also), where it is used to characterize the Hardy spaces associated to the Dunkl operator.

Our purpose is to characterize the local existence of non-tangential boundary limits of $\lambda$-harmonic functions in $\mathbb{R}_{+}^{2}$, which generalizes the theorems of Privalov [38], Marcinkiewicz and Zygmund [30], Spencer [42], Calderón [5,6] and Stein [43]. The main results are stated in the following theorem.

Theorem 1.1. Assume that $u$ is a $\lambda$-harmonic function in $\mathbb{R}_{+}^{2}$, and $E$ is a measurable subset of positive measure of $\partial \mathbb{R}_{+}^{2}=\mathbb{R}$ and is symmetric about $y$-axis. Then the following assertions are equivalent:

(i) $u$ has a finite non-tangential limit at $(x, 0)$ for almost every $x \in E$;

(ii) $u$ is non-tangentially bounded at $(x, 0)$ for almost every $x \in E$;

(iii) the area integral $\left(S_{\alpha, h} u\right)(x)$ is finite for almost every $x \in E$ with some $\alpha, h>0$.

Several remarks are given in order.

(a) For a $\lambda$-harmonic function $u$ in $\mathbb{R}_{+}^{2}$, from $[29,(2.2)]$ we have

$$
\Delta_{\lambda} u^{2}(x, y)=2\left(u_{x}^{2}+u_{y}^{2}\right)+\lambda\left(\frac{u(x, y)-u(-x, y)}{x}\right)^{2},
$$

which implies that $\Delta_{\lambda} u^{2}$ is nonnegative. Further, although the generalized translation operator $\tau_{x}$ is not a positive one, $\left(S_{\alpha, h} u\right)(x)$ preserves positivity since the region $\Gamma_{\alpha}^{h}(0)$ of integration in defining $\left(S_{\alpha, h} u\right)(x)$ is symmetric about $y$-axis. For details, see Section 2.

(b) We note that the assumption of reflection-symmetry on the given subset $E$ of the boundary in Theorem 1.1 is necessary, since the Dunkl operator $D$ involves the value of the function at the reflection-symmetric point.

(c) Here we give a short description on the generalized harmonic functions in the Dunkl setting in the upper half-space $\mathbb{R}_{+}^{d+1}=\mathbb{R}^{d} \times(0, \infty)$ for $d \geq 2$, and the problem on their local non-tangential boundary limits is left for further work.

For given $\lambda_{k} \geq 0, k=1, \cdots, d$, we put $\lambda=\left(\lambda_{1}, \cdots, \lambda_{d}\right)$ as a multiplicity vector. For a differentiable function $f$ on $\mathbb{R}^{d}$, the Dunkl operators are defined by

$$
\mathcal{D}_{k} f(x)=: \frac{\partial}{\partial x_{k}} f(x)+\frac{\lambda_{k}}{x_{k}}\left(f(x)-f\left(\sigma_{k} x\right)\right), \quad k=1, \cdots, d,
$$

where $\sigma_{k} x=\left(x_{1}, \cdots,-x_{k} \cdots, x_{d}\right)$. The associated Laplacian is $\Delta_{\lambda}=\partial_{y}^{2}+\sum_{k=1}^{d} \mathcal{D}_{k^{\prime}}^{2}$ or explicitly, for a twice differentiable function $u$ in $\mathbb{R}_{+}^{d+1}$,

$$
\left(\Delta_{\lambda} u\right)(x, y)=\Delta u(x, y)+\sum_{k=1}^{d} \frac{2 \lambda_{k}}{x_{k}} \frac{\partial}{\partial x_{k}} u(x, y)-\sum_{k=1}^{d} \frac{\lambda_{k}}{x_{k}^{2}}\left(u(x, y)-u\left(\sigma_{k} x, y\right)\right),
$$


where $\Delta=\partial_{y}^{2}+\sum_{k=1}^{d} \partial_{x_{k}}^{2}$ is the usual Laplacian. As above, a $C^{2}$ function $u$ in $\mathbb{R}_{+}^{d+1}$ is said to be $\lambda$-harmonic if $\Delta_{\lambda} u=0$.

(d) The more general setting of the Dunkl theory is on the study of multivariable analytic structures associated with finite reflection groups, of which the basic tools are the Dunkl transform and the Dunkl operators invariant under a given group (cf. [8-13,41,45]). During the last decades, it has gained considerable interest in various fields of mathematics and also in physical applications; for example, the Dunkl operators for the symmetric group $S_{d}$ on $\mathbb{R}^{d}$ are naturally connected with the analysis of quantum many body systems of Calogero-Moser-Sutherland type, which describe algebraically integrable systems in one dimension (cf. [23]).

The paper is organized as follows. Section 2 contains some basic facts on the rank-one case of the Dunkl theory which will be relevant for the sequel. The proof of that (ii) $\Rightarrow$ (i) of Theorem 1.1 is given in Section 3, and the equivalence of parts (ii) and (iii) in Theorem 1.1 is proved in Section 4.

\section{Some facts on the rank-one case of the Dunkl theory}

We denote by $L_{\lambda}^{p}(\mathbb{R})$ the set of measurable functions $f$ on $\mathbb{R}$ satisfying $\|f\|_{L_{\lambda}^{p}}<\infty$, where for $1 \leq p<\infty$,

$$
\|f\|_{L_{\lambda}^{p}}=\left\{c_{\lambda} \int_{\mathbb{R}}|f(x)|^{p}|x|^{2 \lambda} d x\right\}^{1 / p}
$$

with

$$
c_{\lambda}^{-1}=2^{\lambda+1 / 2} \Gamma(\lambda+1 / 2),
$$

and $\|f\|_{L_{\lambda}^{\infty}}=:\|f\|_{\infty}$ is given in the usual way. For sake of simplicity, we set

$$
\langle f, g\rangle_{\lambda}=c_{\lambda} \int_{\mathbb{R}} f(x) g(x)|x|^{2 \lambda} d x
$$

whenever the integral exists, and for a measurable set $E \subset \mathbb{R}$,

$$
|E|_{\lambda}=c_{\lambda} \int_{E}|x|^{2 \lambda} d x \quad \text { and } \quad \sigma E=\{-x: x \in E\} .
$$

$\mathscr{S}(\mathbb{R})$ denotes the space of $C^{\infty}$ functions on $\mathbb{R}$ rapidly decreasing together with their derivatives, and $L_{\lambda, l o c}(\mathbb{R})$ the set of locally integrable functions on $\mathbb{R}$ associated with the measure $|x|^{2 \lambda} d x$. Throughout the paper, the constants $c_{\lambda}, m_{\lambda}, c_{\lambda}^{\prime}$ and $c_{\lambda}^{\prime \prime}$ have always the given values respectively, and $c, c^{\prime}$, and $c^{\prime \prime}$ denote constants which may be different in different occurrences.

For $f \in L_{\lambda}^{1}(\mathbb{R})$, its Dunkl transform is defined by

$$
\left(\mathscr{F}_{\lambda} f\right)(\xi)=c_{\lambda} \int_{\mathbb{R}} f(x) E_{\lambda}(-i x \xi)|x|^{2 \lambda} d x, \quad \xi \in \mathbb{R},
$$


where $E_{\lambda}$ is the Dunkl kernel (cf. [11,40])

$$
E_{\lambda}(z)=j_{\lambda-1 / 2}(i z)+\frac{z}{2 \lambda+1} j_{\lambda+1 / 2}(i z), \quad z \in \mathbb{C},
$$

and $j_{\alpha}(z)$ is the normalized Bessel function

$$
j_{\alpha}(z)=2^{\alpha} \Gamma(\alpha+1) \frac{J_{\alpha}(z)}{z^{\alpha}}=\Gamma(\alpha+1) \sum_{n=0}^{\infty} \frac{(-1)^{n}(z / 2)^{2 n}}{n ! \Gamma(n+\alpha+1)} .
$$

Since $j_{-1 / 2}(z)=\cos z, j_{1 / 2}(z)=z^{-1} \sin z$, it follows that $E_{0}(i z)=e^{i z}$ and $\mathscr{F}_{0}$ agrees with the usual Fourier transform $\mathscr{F}$. In what follows, we assume that $\lambda>0$.

The Dunkl transform shares many of the important properties with the usual Fourier transform, part of which are listed as follows. These conclusions extend those on the Hankel transform and are special cases on the general Dunkl transform studied in $[8,12]$.

Proposition 2.1. (i) If $f \in L_{\lambda}^{1}(\mathbb{R})$, then $\mathscr{F}_{\lambda} f \in C_{0}(\mathbb{R})$ and $\left\|\mathscr{F}_{\lambda} f\right\|_{\infty} \leq\|f\|_{L_{\lambda}^{1}}$.

(ii) (Inversion). If $f \in L_{\lambda}^{1}(\mathbb{R})$ such that $\mathscr{F}_{\lambda} f \in L_{\lambda}^{1}(\mathbb{R})$, then $f(x)=\left[\mathscr{F}_{\lambda}\left(\mathscr{F}_{\lambda} f\right)\right](-x)$.

(iii) For $f \in \mathscr{S}(\mathbb{R})$, we have $\left[\mathscr{F}_{\lambda}(D f)\right](\xi)=i \xi\left(\mathscr{F}_{\lambda} f\right)(\xi),\left[\mathscr{F}_{\lambda}(x f)\right](\xi)=i\left[D_{\tilde{\xi}}\left(\mathscr{F}_{\lambda} f\right)\right](\xi)$ for $\xi \in \mathbb{R}$; and $\mathscr{F}_{\lambda}$ is a topological automorphism on $\mathscr{S}(\mathbb{R})$.

(iv) (Product formula). For all $f, g \in L_{\lambda}^{1}(\mathbb{R})$, we have $\left\langle\mathscr{F}_{\lambda} f, g\right\rangle_{\lambda}=\langle f, \mathscr{F} \lambda g\rangle_{\lambda}$.

(v) (Plancherel). There exists a unique extension of $\mathscr{F}_{\lambda}$ to $L_{\lambda}^{2}(\mathbb{R})$ with $\left\|\mathscr{F}_{\lambda} f\right\|_{L_{\lambda}^{2}}=\|f\|_{L_{\lambda}^{2}}$.

If $(x, t) \neq(0,0)$, the generalized translation $\left(\tau_{t} f\right)(x)$ of $f \in L_{\lambda, l o c}(\mathbb{R})$ associated to the Dunkl transform $\mathscr{F}_{\lambda}$ is defined by (cf. [40])

$$
\left(\tau_{t} f\right)(x)=c_{\lambda}^{\prime} \int_{0}^{\pi}\left(f_{e}\left(\langle x, t\rangle_{\theta}\right)+f_{o}\left(\langle x, t\rangle_{\theta}\right) \frac{x+t}{\langle x, t\rangle_{\theta}}\right)(1+\cos \theta) \sin ^{2 \lambda-1} \theta d \theta
$$

where

$$
\begin{array}{ll}
c_{\lambda}^{\prime}=\Gamma\left(\lambda+\frac{1}{2}\right) /\left(\Gamma(\lambda) \Gamma\left(\frac{1}{2}\right)\right), & f_{e}(x)=\frac{1}{2}(f(x)+f(-x)), \\
f_{o}(x)=\frac{1}{2}(f(x)-f(-x)), & \langle x, t\rangle_{\theta}=\sqrt{x^{2}+t^{2}+2 x t \cos \theta}
\end{array}
$$

If $(x, t)=(0,0)$, we put $\left(\tau_{t} f\right)(x)=f(0)$. We note that $\tau_{t}$ is not a positive operator in general (cf. [40]). An equivalent form of $\tau_{t} f$ for $t \neq 0$ is given by

$$
\left(\tau_{t} f\right)(x)=c_{\lambda} \int_{\mathbb{R}} f(z) W_{\lambda}(x, t, z)|z|^{2 \lambda} d z
$$

where, for $x, t, z \in \mathbb{R}$,

$$
W_{\lambda}(x, t, z)=\frac{c_{\lambda}^{\prime \prime}\left(1-\sigma_{x, t, z}+\sigma_{z, x, t}+\sigma_{z, t, x}\right)|x t z|^{1-2 \lambda}}{\left[\left((|x|+|t|)^{2}-z^{2}\right)\left(z^{2}-(|x|-|t|)^{2}\right)\right]^{1-\lambda}} \chi_{(|| x|-| t|,| x|+| t \mid)}(|z|),
$$


and

$$
\begin{aligned}
& c_{\lambda}^{\prime \prime}=2^{3 / 2-\lambda}(\Gamma(\lambda+1 / 2))^{2} / \sqrt{\pi} \Gamma(\lambda), \\
& \sigma_{x, t, z}=\frac{x^{2}+t^{2}-z^{2}}{2 x t},
\end{aligned}
$$

if $x, t \in \mathbb{R} \backslash\{0\}$, and 0 otherwise.

For two appropriate functions $f$ and $g$ on $\mathbb{R}$, their $\lambda$-convolution $f *_{\lambda} g$ is defined by

$$
\left(f *_{\lambda} g\right)(x)=c_{\lambda} \int_{\mathbb{R}}\left(\tau_{x} f\right)(-t) g(t)|t|^{2 \lambda} d t .
$$

The properties of $\tau_{t}$ and $*_{\lambda}$ are listed as follows (cf. [25,40]).

Proposition 2.2. (i) If $f \in L_{\lambda, l o c}(\mathbb{R})$, then for all $x, t \in \mathbb{R},\left(\tau_{t} f\right)(x)=\left(\tau_{x} f\right)(t)$ and $\left(\tau_{t} \tilde{f}\right)(x)=$ $\left(\widetilde{\tau_{-t} f}\right)(x)$, where $\tilde{f}(x)=f(-x)$.

(ii) If $f \in L_{\lambda, \text { loc }}(\mathbb{R})$ is even and nonnegative, then for all $x, t \in \mathbb{R},\left(\tau_{t} f\right)(x) \geq 0$; and if we define $\tau_{t}^{*}=\left(\tau_{t}+\tau_{-t}\right) / 2$, then for nonnegative $f \in L_{\lambda, \text { loc }}(\mathbb{R})$ and $x, t \in \mathbb{R},\left(\tau_{t}^{*} f\right)(x) \geq 0$.

(iii) For all $1 \leq p \leq \infty$ and $f \in L_{\lambda}^{p}(\mathbb{R}),\left\|\tau_{t} f\right\|_{L_{\lambda}^{p}} \leq 4\|f\|_{L_{\lambda}^{p}}$ with $t \in \mathbb{R}$, and for $1 \leq p<\infty$, $\lim _{t \rightarrow 0}\left\|\tau_{t} f-f\right\|_{L_{\lambda}^{p}}=0$.

(iv) If $f \in L_{\lambda}^{p}(\mathbb{R}), 1 \leq p \leq 2$, and $t \in \mathbb{R}$, then $\left[\mathscr{F}_{\lambda}\left(\tau_{t} f\right)\right](\xi)=E_{\lambda}(i t \xi)\left(\mathscr{F}_{\lambda} f\right)(\xi)$ for almost every $\xi \in \mathbb{R}$.

(v) For measurable $f, g$ on $\mathbb{R}$, we have $\left\langle\tau_{t} f, g\right\rangle_{\lambda}=\left\langle f, \tau_{-t} g\right\rangle_{\lambda}$, whenever the integral

$$
\iint|f(z)||g(x)|\left|W_{\lambda}(x, t, z)\right||z|^{2 \lambda}|x|^{2 \lambda} d z d x
$$

is convergent. In particular, $*_{\lambda}$ is commutative.

(vi) (Young inequality). If $p, q, r \in[1, \infty]$ and $1 / p+1 / q=1+1 / r$, then $\left\|f *_{\lambda} g\right\|_{L_{\lambda}^{r}} \leq$

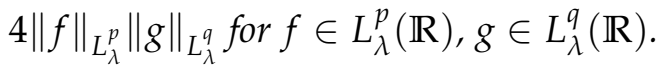

(vii) Assume that $p, q, r \in[1,2]$ and $1 / p+1 / q=1+1 / r$. Then for $f \in L_{\lambda}^{p}(\mathbb{R}), g \in$ $L_{\lambda}^{q}(\mathbb{R}),\left[\mathscr{F}_{\lambda}\left(f *_{\lambda} g\right)\right](\xi)=\left(\mathscr{F}_{\lambda} f\right)(\xi)(\mathscr{F} \lambda g)(\xi)$. In particular $*_{\lambda}$ is associative in $L_{\lambda}^{1}(\mathbb{R})$.

The $\lambda$-Poisson integral of $f \in L_{\lambda}^{p}(\mathbb{R}), 1 \leq p \leq \infty$, is defined by $(P f)(x, y)=(f * \lambda$ $\left.P_{y}\right)(x)$, i.e.,

$$
(P f)(x, y)=c_{\lambda} \int_{\mathbb{R}} f(t)\left(\tau_{x} P_{y}\right)(-t)|t|^{2 \lambda} d t, \quad(x, y) \in \mathbb{R}_{+}^{2},
$$

where

$$
P_{y}(x)=m_{\lambda} y\left(y^{2}+x^{2}\right)^{-\lambda-1}
$$

is the $\lambda$-Poisson kernel and

$$
m_{\lambda}=2^{\lambda+1 / 2} \Gamma(\lambda+1) / \sqrt{\pi}
$$

For $\alpha>0$, the non-tangential maximal function is

$$
\left(P_{\nabla}^{*} f\right)(x)=\sup _{(t, y) \in \Gamma_{\alpha}(x)}|(P f)(t, y)| .
$$


Proposition 2.3 (Theorem 5.4 in [45]). For $f \in L_{\lambda}^{p}(\mathbb{R}), 1 \leq p \leq \infty,\|(P f)(\cdot, y)\|_{L_{\lambda}^{p}} \leq\|f\|_{L_{\lambda}^{p} \text {; }}$ and for $f \in X=L_{\lambda}^{p}(\mathbb{R}), 1 \leq p<\infty$, or $C_{0}(\mathbb{R}), \lim _{y \rightarrow 0+}\|(P f)(\cdot, y)-f\|_{X}=0$.

Proposition 2.4 (Theorem 3.8 and Corollary 3.9 in [25]). (i) The non-tangential maximal operator $P_{\nabla}^{*}$ is of type $(p, p)$ for $1<p \leq \infty$ and of weak-type $(1,1)$.

(ii) Assume that $\alpha>0$. If $f \in L_{\lambda}^{p}(\mathbb{R}), 1 \leq p \leq \infty$, then for almost every $x \in \mathbb{R}$, the $\lambda$-Poisson integral $(P f)(t, y)$ converges to $f(x)$ as $(t, y) \in \Gamma_{\alpha}(x)$ approaching to $(x, 0)$.

The Green formula and the maximum principle associated with the Dunkl operator are given in the following propositions.

Proposition 2.5 (Green's formula, (38) in [25]). Assume that the bounded domain $\Omega \subset \mathbb{R}^{2}$ is symmetric about $y$-axis and with piecewise-smooth boundary curve $\partial \Omega$. Then for $u, v \in C^{2}(\bar{\Omega})$,

$$
\iint_{\Omega}\left(v \Delta_{\lambda} u-u \Delta_{\lambda} v\right)|x|^{2 \lambda} d x d y=\int_{\partial \Omega}|x|^{2 \lambda}\left(v \frac{\partial u}{\partial \mathbf{n}}-u \frac{\partial v}{\partial \mathbf{n}}\right) d \ell,
$$

where $\partial / \partial \mathbf{n}$ denotes the directional derivative of the outward normal.

Proposition 2.6 (Maximum Principle, Lemma 3.2 in [26]). Let $\Omega \subseteq \mathbb{R}^{2}$ be an open bounded region symmetric about $y$-axis, and let $u \in C(\bar{\Omega})$. Assume that $u$ is of class $C^{2}$ in the region where $u>0$ and satisfies $\Delta_{\lambda} u \geq 0$ there. If $\left.u\right|_{\partial \Omega} \leq 0$, then $u \leq 0$ on the whole $\Omega$.

As a corollary we have

Proposition 2.7 (Maximum Principle). Let $\Omega \subset \mathbb{R}^{2}$ be an open bounded domain symmetric about $y$-axis, and let $u \in C(\bar{\Omega})$. If $u$ is $\lambda$-harmonic in $\Omega$, then

$$
\max _{(x, y) \in \bar{\Omega}} u(x, y)=\max _{(x, y) \in \partial \Omega} u(x, y) .
$$

\section{The proof of the main theorem}

In this section, we shall prove that (ii) $\Rightarrow$ (i) in Theorem 1.1, which is restated as follows.

Theorem 3.1. Assume that $u$ is a $\lambda$-harmonic function in $\mathbb{R}_{+}^{2}$, and $E$ is a measurable set of positive measure of $\partial \mathbb{R}_{+}^{2}=\mathbb{R}$ and is symmetric about $y$-axis. If $u$ is non-tangentially bounded at $(x, 0)$ for every $x \in E$, then $u$ has a finite non-tangential limit at $(x, 0)$ for almost every $x \in E$.

It is noted that, in the above theorem, we are only assuming that $u$ is bounded in $\Gamma_{\alpha}^{h}(x)$ for some $\alpha, h>0$, and in particular $\alpha, h$ can depend on $x$. Nevertheless, the conclusion is that there exists a subset $E_{0}$ of full measure of $E$ such that for every $\alpha>0$ and each $x \in E_{0}, u(t, y)$ has a finite limit as $(t, y) \in \Gamma_{\alpha}(x)$ approaches to $(x, 0)$. The reasons for this is partly contained in the following lemma. For a subset $E$ of $\partial \mathbb{R}_{+}^{2}=\mathbb{R}$ and for fixed $\alpha, h>0$, we always use the notation

$$
\Omega^{E}(\alpha, h)=: \bigcup_{x_{0} \in E} \Gamma_{\alpha}^{h}\left(x_{0}\right) .
$$


Lemma 3.1 (pp. 201 in [44]). Let $u$ be a continuous function in $\mathbb{R}_{+}^{2}$, and $E$ a measurable set of $\partial \mathbb{R}_{+}^{2}=\mathbb{R}$ with $0<|E|_{0}<\infty$, where $|\cdot|_{0}$ denotes the Lebesgue measure. If $u$ is non-tangentially bounded at $(x, 0)$ for every $x \in E$, then for any $\epsilon>0$, there exists a compact set $E_{1}$ satisfying

(i) $E_{1} \subset E,\left|E-E_{1}\right|_{0}<\epsilon$;

(ii) for any $\alpha>0$ and $h>0$, there is a constant $c_{\alpha, h, \epsilon}>0$, so that

$$
|u(x, y)| \leq c_{\alpha, h, \epsilon,} \quad(x, y) \in \Omega^{E_{1}}(\alpha, h) .
$$

Lemma 3.2. If $u$ is a $\lambda$-harmonic function in $\mathbb{R}_{+}^{2}$, then $\Delta_{\lambda}|u| \geq 0$ in the region where $u \neq 0$.

Indeed, a direct calculation shows that

$$
\Delta_{\lambda}|u(x, y)|=\frac{\lambda}{x^{2}}[|u(-x, y)|-(\operatorname{sgn} u(x, y)) u(-x, y)] \geq 0 .
$$

The next lemma plays a crucial role in the proof of Theorem 3.1.

Lemma 3.3. Let $E$ be a measurable set of $\partial \mathbb{R}_{+}^{2}=\mathbb{R}$, symmetric about $y$-axis, and for $\alpha>0$, $\Omega=\Omega^{E}(\alpha, 1)$. Then there exists a nonnegative $\lambda$-harmonic function $H$ in $\mathbb{R}_{+}^{2}$ satisfying

(i) $H(x, y)$ is even in $x$ and $H(x, y) \geq 2$ for $(x, y) \in \mathbb{R}_{+}^{2} \cap \partial \Omega$;

(ii) $H$ has non-tangential limit 0 at $(x, 0)$ for almost every $x \in E$.

Proof. We first define

$$
H_{0}(x, y)=\left(P \chi_{E^{c}}\right)(x, y)+y,
$$

where $\chi_{E^{c}}$ is the characteristic function of the complement $E^{c}$ of $E$. It is obvious that $H_{0}$ is nonnegative and $\lambda$-harmonic in $\mathbb{R}_{+}^{2}$, and also even in $x$ since $\chi_{E^{c}}$ is even. By Proposition 2.4, $H$ has non-tangential limit 0 at $(x, 0)$ for almost every $x \in E$.

Now we prove that $H_{0}$ has a positive lower bound on $\mathbb{R}_{+}^{2} \cap \partial \Omega$. For $(x, y) \in \partial \Omega$ with $y=1, H(x, y) \geq 1$. If $(x, y) \in \partial \Omega$ with $0<y<1$, then $\{t:|t-x|<\alpha y\} \subset E^{c}$; this is because, $t^{\prime} \in E$ with $\left|t^{\prime}-x\right|<\alpha y$ implies that $(x, y) \in \Gamma_{\alpha}^{1}\left(t^{\prime}\right) \subset \Omega$. Thus, by (2.3) we have

$$
H_{0}(x, y) \geq c_{\lambda} \int_{|t-x|<\alpha y} \tau_{x} P_{y}(-t)|t|^{2 \lambda} d t
$$

We need to use the following estimate for the $\lambda$-Poisson kernel $\tau_{x} P_{y}(-t)$ (cf. [25, Corollary 3.7])

$$
\left(\tau_{x} P_{y}\right)(-t) \asymp \frac{y[y+|x|+|t|]^{-2 \lambda}}{y^{2}+(x-t)^{2}} \ln \left(\frac{y^{2}+(x-t)^{2}}{y^{2}+(x+t)^{2}}+2\right) .
$$

Since, for $|t-x|<\alpha y$,

$$
y^{2}+(x-t)^{2} \leq\left(\alpha^{2}+1\right) y^{2} \text { and } y+|x|+|t| \leq(\alpha+1) y+2|x|,
$$


it follows that, for some $c>0$,

$$
\left(\tau_{x} P_{y}\right)(-t) \geq c y^{-1}(y+|x|)^{-2 \lambda} .
$$

Then from (3.1) we have

$$
H_{0}(x, h) \geq \frac{c}{y(y+|x|)^{2 \lambda}} \int_{|t-x|<\alpha y}|t|^{2 \lambda} d t
$$

and since

$$
\int_{|t-x|<\alpha y}|t|^{2 \lambda} d t \asymp y(|x|+y)^{2 \lambda}
$$

we conclude that $H_{0}$ has a positive lower bound $c_{0}$ on $\mathbb{R}_{+}^{2} \cap \partial \Omega$. Finally the function $H=2 H_{0} / c_{0}$ is desired.

Lemma 3.4. Let $u(x, y)$ be a $\lambda$-harmonic function in $\mathbb{R}_{+}^{2}, E$ a compact subset of $\partial \mathbb{R}_{+}^{2}=\mathbb{R}$ being symmetric about $y$-axis, and for $\alpha>0, \Omega=\Omega^{E}(\alpha, 2)$. If

$$
|u(x, y)| \leq 1, \quad(x, y) \in \Omega,
$$

then for almost every $x \in E, u(t, y)$ has a finite limit as $(t, y) \in \Gamma_{\alpha}(x)$ approaching to $(x, 0)$.

Proof. We first note that $\Omega$ is an open bounded domain in $\mathbb{R}_{+}^{2}$, symmetric about $y$-axis. Choose a sequence $\left\{y_{k}\right\}_{k=1}^{\infty} \subset(0,1)$ such that $y_{k} \rightarrow 0$, and for $x \in \mathbb{R}$, define

$$
\varphi_{k}(x)= \begin{cases}u\left(x, y_{k}\right), & \text { if }\left(x, y_{k}\right) \in \Omega, \\ 0, & \text { otherwise. }\end{cases}
$$

Obviously $\left|\varphi_{k}\right| \leq 1$ on $\mathbb{R}$ for all $k \geq 1$, and thus, one can find a function $\varphi$ with $|\varphi| \leq 1$ on $\mathbb{R}$ and a subsequence $\left\{\varphi_{k_{j}}\right\}$ so that $\left\{\varphi_{k_{j}}\right\}$ converges weakly ${ }^{*}$ to $\varphi$. In particular, for their $\lambda$-Poisson integrals, we have

$$
\lim _{j \rightarrow \infty}\left(P \varphi_{k_{j}}\right)(x, y)=(P \varphi)(x, y), \quad(x, y) \in \mathbb{R}_{+}^{2} .
$$

If we write

$$
\psi_{k}(x, y)=u\left(x, y+y_{k}\right)-\left(P \varphi_{k}\right)(x, y)
$$

then

$$
\psi(x, y)=: \lim _{j \rightarrow \infty} \psi_{k_{j}}(x, y)=u(x, y)-(P \varphi)(x, y), \quad(x, y) \in \mathbb{R}_{+}^{2} .
$$

Further we claim that, for each $k$,

$$
\left|\psi_{k}(x, y)\right| \leq 2 H(x, y), \quad(x, y) \in \Omega_{1}=: \Omega^{E}(\alpha, 1),
$$


where $H$ is the function given in Lemma 3.3. In fact, we shall prove the following stronger version of (3.6)

$$
\left.\left|\psi_{k}(x, y)\right|+\mid \psi_{k}(-x, y)\right) \mid \leq 2 H(x, y), \quad(x, y) \in \Omega_{1} .
$$

If there were some $\left(x_{0}, y_{0}\right) \in \Omega_{1}$ so that $\left|\psi_{k}\left(x_{0}, y_{0}\right)\right|+\left|\psi_{k}\left(-x_{0}, y_{0}\right)\right|>2 H\left(x_{0}, y_{0}\right)$, we consider the set

$$
G=\left\{(x, y) \in \Omega_{1}: \Psi_{k}(x, y)-2 H(x, y)>\epsilon_{0}\right\},
$$

where

$$
\left.\Psi_{k}(x, y)=\mid \psi_{k}(x, y) \pm \psi_{k}(-x, y)\right) \mid \quad \text { and } \quad \epsilon_{0}=\frac{1}{2} \Psi_{k}\left(x_{0}, y_{0}\right)-H\left(x_{0}, y_{0}\right) .
$$

The choice of plus or minus sign depends on

$$
\psi_{k}\left(x_{0}, y_{0}\right) \psi_{k}\left(-x_{0}, y_{0}\right) \geq 0 \quad \text { or } \quad \psi_{k}\left(x_{0}, y_{0}\right) \psi_{k}\left(-x_{0}, y_{0}\right) \leq 0 \text {. }
$$

It follows that the set $G$ is non-empty, open and symmetric about $y$-axis. By Lemma 3.2 and Proposition 2.6 (the maximum principle), we have $\partial G \cap \partial \Omega_{1} \neq \varnothing$, since, otherwise, $\bar{G} \subset \Omega_{1}$ and the function $\Psi_{k}-2 H$ would attain its maximum value inside $\Omega_{1}$.

For $\left(x^{*}, y^{*}\right) \in \partial G \cap \partial \Omega_{1}$, there exists a sequence of points $\left\{\left(\hat{x}_{\ell}, \hat{y}_{\ell}\right)\right\} \subset G$ converging to $\left(x^{*}, y^{*}\right)$, so that

$$
2 H\left(\hat{x}_{\ell}, \hat{y}_{\ell}\right)+\epsilon_{0}<\Psi_{k}\left(\hat{x}_{\ell}, \hat{y}_{\ell}\right), \quad \ell=1, \cdots .
$$

If $y^{*}>0$, letting $\ell \rightarrow \infty$, gives

$$
2 H\left(x^{*}, y^{*}\right)+\epsilon_{0} \leq \Psi_{k}\left(x^{*}, y^{*}\right) ;
$$

but by Proposition 2.3 and (3.4),

$$
\left|\psi_{k}\left( \pm x^{*}, y^{*}\right)\right| \leq\left|u\left( \pm x^{*}, y^{*}+y_{k}\right)\right|+\left|\left(P \varphi_{k}\right)\left( \pm x^{*}, y^{*}\right)\right| \leq 2,
$$

which implies $\Psi_{k}\left(x^{*}, y^{*}\right) \leq 4$ and leads to a contradiction to the fact $H\left(x^{*}, y^{*}\right) \geq 2$ by Lemma 3.3. Hence $y^{*}=0$, and further $\pm x^{*} \in E$.

Since $\varphi_{k}(x)=u\left(x, y_{k}\right)$ for $\left|x-x^{*}\right|<\alpha y_{k}, \varphi_{k}$ is continuous at $x^{*}$. Now from (2.3) and (3.2), we have

$$
\begin{aligned}
& \left|\left(P \varphi_{k}\right)\left(\hat{x}_{\ell}, \hat{y}_{\ell}\right)-\varphi_{k}\left(x^{*}\right)\right| \\
\leq & c_{\lambda} \int_{\mathbb{R}}\left|\varphi_{k}(t)-\varphi_{k}\left(x^{*}\right)\right|\left(\tau_{\hat{x}_{\ell}} P_{\hat{y}_{\ell}}\right)(-t)|t|^{2 \lambda} d t \\
\leq & c \int_{\mathbb{R}}\left|\varphi_{k}(t)-\varphi_{k}\left(x^{*}\right)\right| \frac{\hat{y}_{\ell}}{\hat{y}_{\ell}^{2}+\left(\hat{x}_{\ell}-t\right)^{2}} \ln \left(\frac{\left(\hat{x}_{\ell}-t\right)^{2}}{\hat{y}_{\ell}^{2}}+3\right) d t \\
= & c \int_{\mathbb{R}}\left|\varphi_{k}\left(\hat{x}_{\ell}-\hat{y}_{\ell} t\right)-\varphi_{k}\left(x^{*}\right)\right| \frac{\ln \left(t^{2}+3\right)}{1+t^{2}} d t,
\end{aligned}
$$


and by the Lebesgue dominated convergence theorem, $\left(P \varphi_{k}\right)\left(\hat{x}_{\ell}, \hat{y}_{\ell}\right)$ tends to $\varphi_{k}\left(x^{*}\right)$ as $\ell \rightarrow \infty$. It follows that

$$
\lim _{\ell \rightarrow \infty} \psi_{k}\left(\hat{x}_{\ell}, \hat{y}_{\ell}\right)=u\left(x^{*}, y_{k}\right)-\varphi_{k}\left(x^{*}\right)=0
$$

and similarly

$$
\lim _{\ell \rightarrow \infty} \psi_{k}\left(-\hat{x}_{\ell}, \hat{y}_{\ell}\right)=0
$$

Thus, from (3.8) we have

$$
\limsup _{\ell \rightarrow \infty} H\left(\hat{x}_{\ell}, \hat{y}_{\ell}\right) \leq-\epsilon_{0},
$$

which contradicts the nonnegativity of $H$ by Lemma 3.3. The claim (3.7), and so (3.6), are proved.

Finally, taking $k=k_{j}$ in (3.6) and letting $j \rightarrow \infty$ yields $|\psi(x, y)| \leq 2 H(x, y)$ for $(x, y) \in$ $\Omega_{1}$, and by Lemma 3.3, for almost every $x \in E, \psi(t, y)$ tends to zero as $(t, y) \in \Gamma_{\alpha}(x)$ approaching to $(x, 0)$. Further by Proposition 2.4, $(P \varphi)(x, y)$ has a finite non-tangential limit for almost every $x \in E$, and hence, by (3.5), $u(x, y)=\psi(x, y)+(P \varphi)(x, y)$ has the desired assertion in the lemma.

Now we turn to the proof of Theorem 3.1:

Proof. We assume that the set $E$ is bounded, without loss of generality. By Lemma 3.1, for each $k \in \mathbb{N}$, there exists a compact set $E_{k} \subset E$, such that $\left|E \backslash E_{k}\right|_{0}<1 / k$, and for any $\alpha>0$, there is a constant $c_{\alpha, k}>0$, so that $|u(x, y)| \leq c_{\alpha, k},(x, y) \in \bigcup_{x_{0} \in E_{k}} \Gamma_{\alpha}^{2}\left(x_{0}\right)$. If we put $E_{0}=\bigcup_{k=1}^{\infty} E_{k}$, then $\left|E \backslash E_{0}\right|_{0}=0$. Since $E$ is symmetric about $y$-axis, we may choose each $E_{k}$ preserving this property; and otherwise, $E_{k} \cap \sigma E_{k}$ could be used instead of $E_{k}$. Thus applying Lemma 3.4 to $u / c_{\alpha, k}$, it follows that, for almost every $x \in E_{k}, u(t, y) / c_{\alpha, k}$ has a finite limit as $(t, y) \in \Gamma_{\alpha}(x)$ approaching to $(x, 0)$, and hence, $u$ has a finite non-tangential limit at $(x, 0)$ for almost every $x \in E$. The proof is completed.

\section{The proof of the main theorem (continue)}

We recall that, for a $C^{2}$ function $u$ in $\mathbb{R}_{+}^{2}$, the Lusin-type area integral $S u=S_{\alpha, h} u$ for some $\alpha, h>0$ is defined by

$$
\left(S_{\alpha, h} u\right)(x)=\left(\int_{\Gamma_{\alpha}^{h}(0)} \tau_{x}\left(\Delta_{\lambda} u^{2}\right)(-t, y) y^{-2 \lambda}|t|^{2 \lambda} d t d y\right)^{1 / 2} .
$$

In this section, we shall prove the equivalence of parts (ii) and (iii) in Theorem 1.1, which is reformulated in the following two theorems.

Theorem 4.1. Assume that $u$ is a $\lambda$-harmonic function in $\mathbb{R}_{+}^{2}$, and $E$ is a measurable subset of positive measure of $\partial \mathbb{R}_{+}^{2}=\mathbb{R}$ and is symmetric about $y$-axis. If $u$ is non-tangentially bounded at $(x, 0)$ for every $x \in E$, then for arbitrary $\alpha, h>0$, the area integral $\left(S_{\alpha, h} u\right)(x)$ is finite for almost every $x \in E$. 
Theorem 4.2. Assume that $u$ is a $\lambda$-harmonic function in $\mathbb{R}_{+}^{2}$, and $E$ is a measurable subset of positive measure of $\partial \mathbb{R}_{+}^{2}=\mathbb{R}$ and is symmetric about $y$-axis. If for every $x \in E$, there exist some $\alpha, h>0$, so that the area integral $\left(S_{\alpha, h} u\right)(x)$ is finite, then $u$ is non-tangentially bounded at $(x, 0)$ for almost every $x \in E$.

Clearly Theorem 4.1 is a stronger version of that (ii) $\Rightarrow$ (iii) in Theorem 1.1. To prove Theorems 4.1 and 4.2, we need several lemmas.

Lemma 4.1. Let $E$ be a measurable and bounded set of $\partial \mathbb{R}_{+}^{2}=\mathbb{R}$, symmetric about $y$-axis. Then for any $\epsilon>0$, there exists a compact set $E_{\epsilon}$, symmetric about $y$-axis, satisfying

(i) $E_{\epsilon} \subset E,\left|E-E_{\epsilon}\right|_{\lambda}<\epsilon$;

(ii) for $\eta \in(0,1)$, there exists some $\delta>0$, such that for $x \in E_{\epsilon}$ and $0<r<\delta$,

$$
|(x-r, x+r) \cap E|_{\lambda}>\eta|(x-r, x+r)|_{\lambda} .
$$

Proof. For $f \in L_{\lambda, l o c}(\mathbb{R})$, we define the weighted maximal function $M_{\lambda} f$ by

$$
\left(M_{\lambda} f\right)(x)=\sup _{r>0} c_{\lambda} \int_{x-r}^{x+r}|f(t)||t|^{2 \lambda} d t /|(x-r, x+r)|_{\lambda} .
$$

Since $M_{\lambda}$ is of weak-type $(1,1)$, by a standard process one can prove that

$$
\lim _{r \rightarrow 0+} c_{\lambda} \int_{x-r}^{x+r}|f(t)||t|^{2 \lambda} d t /|(x-r, x+r)|_{\lambda}=f(x)
$$

for a.e. $x \in \mathbb{R}$.

Now taking $f=\chi_{E}$, it follows that

$$
\lim _{r \rightarrow 0+} \frac{|(x-r, x+r) \cap E|_{\lambda}}{|(x-r, x+r)|_{\lambda}}=1 \quad \text { for a.e. } x \in E \text {. }
$$

By Egorov's theorem, for given $\epsilon>0$ there exists $E_{\epsilon} \subset E,\left|E-E_{\epsilon}\right|_{\lambda}<\epsilon$, so that $|(x-r, x+r) \cap E|_{\lambda} /|(x-r, x+r)|_{\lambda}$ tends to 1 uniformly for $x \in E_{\epsilon}$ as $r \rightarrow 0+$. Clearly one may take $E_{\epsilon}$ to be a closed subset of $E$. Since $E$ is symmetric about $y$-axis, the uniform convergence above is true also for $x \in \sigma E_{\epsilon}$, and so is for $x \in E_{\epsilon} \cup \sigma E_{\epsilon}$. Thus we may take $E_{\epsilon}$ to be symmetric about $y$-axis, as desired.

Lemma 4.2. Let $u(x, y)$ be a $\lambda$-harmonic function in $\mathbb{R}_{+}^{2}, E$ a compact subset of $\partial \mathbb{R}_{+}^{2}=\mathbb{R}$ being symmetric about $y$-axis, and for $\alpha, h>0, \Omega=\Omega^{E}(\alpha, h)$. Then

$$
\int_{E}\left(S_{\alpha, h} u\right)^{2}(x)|x|^{2 \lambda} d x \leq c \iint_{\Omega} y\left(\Delta_{\lambda} u^{2}\right)(t, y)|t|^{2 \lambda} d t d y,
$$

whenever the right hand side above is finite. 
Proof. Let $\chi_{\Gamma_{\alpha}^{h}(0)}$ be the characteristic function of $\Gamma_{\alpha}^{h}(0)$, i.e.,

$$
\chi_{\Gamma_{\alpha}^{h}(0)}(x, y)=1 \text { if }|x|<\alpha y \text { and } 0<y<h,
$$

and $\chi_{\Gamma_{\alpha}^{h}(0)}(x, y)=0$ otherwise. By Proposition 2.2(i) and (v), we have

$$
\begin{aligned}
& \int_{E}\left(S_{\alpha, h} u\right)^{2}(x)|x|^{2 \lambda} d x \\
= & \int_{E} \iint_{\mathbb{R}_{+}^{2}}\left(\Delta_{\lambda} u^{2}\right)(-t, y)\left(\tau_{x} \chi_{\Gamma_{\alpha}^{h}(0)}\right)(t, y) y^{-2 \lambda}|t|^{2 \lambda} d t d y|x|^{2 \lambda} d x,
\end{aligned}
$$

where $\tau_{x}$ acts on the first argument of $\chi_{\Gamma_{\alpha}^{h}(0)}$. If for $x \in E,(t, y) \notin \Gamma_{\alpha}^{h}(x) \cup \Gamma_{\alpha}^{h}(-x)$, then

$$
y \geq h \quad \text { or } \quad 0<y<h, \quad \text { with }|| t|-| x|| \geq \alpha y,
$$

and in the later case, the translation kernel (see (2.2)) $W_{\lambda}(x, t, z)$ vanishes for $(z, y) \in$ $\Gamma_{\alpha}^{h}(0)$. Thus we have

$$
\left(\tau_{x} \chi_{\Gamma_{\alpha}^{h}(0)}\right)(t, y)=0, \quad(t, y) \notin \Gamma_{\alpha}^{h}(x) \cup \Gamma_{\alpha}^{h}(-x),
$$

and then, in view of the symmetry of $E$ about $y$-axis,

$$
\int_{E}\left(S_{\alpha, h} u\right)^{2}(x)|x|^{2 \lambda} d x \leq \iint_{\Omega}\left(\Delta_{\lambda} u^{2}\right)(-t, y) k_{1}(t, y) y^{-2 \lambda}|t|^{2 \lambda} d t d y
$$

where

$$
k_{1}(t, y)=\int_{E}\left(\tau_{x} \chi_{\Gamma_{\alpha}^{h}(0)}\right)(t, y)|x|^{2 \lambda} d x
$$

By Proposition 2.2(i), (ii) and (iii), it follows that

$$
\begin{aligned}
k_{1}(t, y) & =\int_{E}\left(\tau_{t} \chi_{\Gamma_{\alpha}^{h}(0)}\right)(x, y)|x|^{2 \lambda} d x \leq \int_{\mathbb{R}}\left(\tau_{t} \chi_{(-\alpha y, \alpha y)}\right)(x)|x|^{2 \lambda} d x \\
& \leq 4 \int_{\mathbb{R}} \chi_{(-\alpha y, \alpha y)}(x)|x|^{2 \lambda} d x=c y^{2 \lambda+1} .
\end{aligned}
$$

Substituting this into (4.4) and in consideration of the symmetry of $\Omega$ about $y$-axis, (4.1) is proved.

Lemma 4.3. Let $u(x, y)$ be a $\lambda$-harmonic function in $\mathbb{R}_{+}^{2}, E$ a measurable and bounded set of $\partial \mathbb{R}_{+}^{2}=\mathbb{R}$ being symmetric about $y$-axis, and let $\beta, \kappa>0$ be given. Then for any $\epsilon>0$, there exists a compact set $E_{\epsilon}$, symmetric about $y$-axis, satisfying

(i) $E_{\epsilon} \subset E,\left|E-E_{\epsilon}\right|_{\lambda}<\epsilon$;

(ii) for fixed $\alpha \in(0, \beta)$ and $h \in(0, \kappa)$, there exists some $c=c(\epsilon, \alpha, \beta, h, \kappa)>0$, such that

$$
\iint_{\Omega^{E_{E}(\alpha, h)}} y\left(\Delta_{\lambda} u^{2}\right)(t, y)|t|^{2 \lambda} d t d y \leq c \int_{E}\left(S_{\beta, \kappa} u\right)^{2}(x)|x|^{2 \lambda} d x,
$$

whenever the right hand side above is finite. 
Proof. For given $\epsilon>0$, by Lemma 4.1 there exist a compact subset $E_{\epsilon}$ of $E$, symmetric about $y$-axis, and some $\delta>0$, satisfying $\left|E-E_{\epsilon}\right|_{\lambda}<\epsilon$ and

$$
|(x-r, x+r) \cap E|_{\lambda}>\frac{1}{2}|(x-r, x+r)|_{\lambda}
$$

for $x \in E_{\epsilon}$ and $0<r<\delta$.

Now we fix $\alpha<\beta$ and $h<\kappa$. Since $(t, y) \in \Gamma_{\beta}^{\kappa}(x) \cup \Gamma_{\beta}^{\kappa}(-x)$ is equivalent to $(x, y) \in$ $\Gamma_{\beta}^{\kappa}(t) \cup \Gamma_{\beta}^{\kappa}(-t)$, from (4.2) and (4.3) and by Proposition 2.2(i), we have

$$
\int_{E}\left(S_{\beta, \kappa} u\right)^{2}(x)|x|^{2 \lambda} d x=\iint_{\Omega^{E}(\beta, \kappa)}\left(\Delta_{\lambda} u^{2}\right)(t, y) k_{2}(t, y) y^{-2 \lambda}|t|^{2 \lambda} d t d y
$$

where

$$
k_{2}(t, y)=\int_{E}\left(\tau_{-t} \chi_{\Gamma_{\beta}^{\kappa}(0)}\right)(x, y) \chi_{\Gamma_{\beta}^{\kappa}(t) \cup \Gamma_{\beta}^{\kappa}(-t)}(x, y)|x|^{2 \lambda} d x
$$

For given $(t, y) \in \Omega^{E_{\epsilon}}(\alpha, h)$, there exists some $\bar{x} \in E_{\epsilon}$ such that $(t, y) \in \Gamma_{\alpha}^{h}(\bar{x})$. Thus, when

$$
|x-\bar{x}|<\gamma y, \quad \gamma=\min \{(\beta-\alpha) / 2, \delta / h\},
$$

we have $|x-t|<\alpha^{\prime} y$ with $\alpha^{\prime}=(\alpha+\beta) / 2$, which, certainly, implies that $(x, y) \in \Gamma_{\beta}^{\kappa}(t)$.

We claim that, there exists a constant $c=c(\alpha, \beta)>0$, such that for $(x, y) \in \Gamma_{\alpha^{\prime}}^{h}(t)$,

$$
\left(\tau_{-t} \chi_{\Gamma_{\beta}^{\kappa}(0)}\right)(x, y) \geq c\left(\frac{y^{2}}{|x t|+y^{2}}\right)^{\lambda}
$$

In fact, from (2.1) it follows that

$$
\left(\tau_{-t} \chi_{\Gamma_{\beta}^{\kappa}(0)}\right)(x, y)=c_{\lambda}^{\prime} \int_{-1}^{1} \chi_{\left\{s: x^{2}+t^{2}-2 x t s<\beta^{2} y^{2}\right\}}(s)(1+s)^{\lambda}(1-s)^{\lambda-1} d s ;
$$

but for

$$
x^{2}+t^{2}-2 x t s=(x-t)^{2}+2 x t(1-s) \leq(x-t)^{2}+2|x t|(1-s),
$$

we have

$$
\left(\tau_{-t} \chi_{\Gamma_{\beta}^{\kappa}(0)}\right)(x, y) \geq c_{\lambda}^{\prime} \int_{0}^{1} \chi_{\left\{s:(x-t)^{2}+2|x t|(1-s)<\beta^{2} y^{2}\right\}}(s)(1-s)^{\lambda-1} d s .
$$

If $(x-t)^{2}+2|x t|<\beta^{2} y^{2}$, then

$$
\left(\tau_{-t} \chi_{\Gamma_{\beta}^{\kappa}(0)}\right)(x, y) \geq c_{\lambda} \int_{0}^{1}(1-s)^{\lambda-1} d s=c,
$$


which concludes (4.9); if $(x-t)^{2}+2|x t| \geq \beta^{2} y^{2}$, one has

$$
\begin{aligned}
\left(\tau_{-t} \chi_{\Gamma_{\beta}^{\kappa}(0)}\right)(x, y) & \geq c_{\lambda}^{\prime} \int_{1-\frac{\beta^{2} y^{2}-(x-t)^{2}}{2|x t|}}^{1}(1-s)^{\lambda-1} d s \\
& =c\left(\frac{\beta^{2} y^{2}-(x-t)^{2}}{2|x t|}\right)^{\lambda},
\end{aligned}
$$

and so, for $(x, y) \in \Gamma_{\alpha^{\prime}}^{h}(t)$,

$$
\left(\tau_{-t} \chi_{\Gamma_{\beta}^{\kappa}(0)}\right)(x, y) \geq c^{\prime}\left(y^{2} /|x t|\right)^{\lambda}
$$

which again concludes (4.9).

Now we show that, for $x$ satisfying (4.8),

$$
|x t|+y^{2} \asymp|\bar{x}|^{2}+y^{2} \text {. }
$$

If $|t| \leq 2 \beta y$, then

$$
\begin{aligned}
& |\bar{x}| \leq|\bar{x}-t|+|t| \leq \alpha y+2 \beta y<3 \beta y, \\
& |x| \leq|x-\bar{x}|+|\bar{x}|<4 \beta y,
\end{aligned}
$$

thus (4.10) is verified. If $|t|>2 \beta y$, we have

$$
\begin{aligned}
& |\bar{x}| \leq|t|+|\bar{x}-t| \leq|t|+\alpha y<2|t|, \\
& |\bar{x}| \geq|t|-|\bar{x}-t| \geq|t|-\alpha y \geq|t| / 2, \\
& |x| \leq|\bar{x}|+|x-\bar{x}| \leq|\bar{x}|+\beta y \leq|\bar{x}|+|t| / 2 \leq 2|\bar{x}|, \\
& |x| \geq|\bar{x}|-|x-\bar{x}| \geq|\bar{x}|-\beta y / 2 \geq|\bar{x}|-|t| / 4 \geq|\bar{x}| / 2,
\end{aligned}
$$

and then, collecting these estimates verifies (4.10) again.

Applying (4.9) and (4.10) to (4.7) we obtain, for $(t, y) \in \Omega^{E_{\epsilon}}(\alpha, h)$,

$$
k_{2}(t, y) \geq c\left(\frac{y}{|\bar{x}|+y}\right)^{2 \lambda}|(\bar{x}-\gamma y, \bar{x}+\gamma y) \cap E|_{\lambda} ;
$$

and for $\bar{x} \in E_{\epsilon}$ and $\gamma y \leq \delta y / h<\delta$, appealing to (4.5) and the estimate

$$
|(\bar{x}-\gamma y, \bar{x}+\gamma y)|_{\lambda} \asymp y(|\bar{x}|+y)^{2 \lambda}
$$

yields

$$
k_{2}(t, y) \geq \frac{c}{2}\left(\frac{y}{|\bar{x}|+y}\right)^{2 \lambda}|(\bar{x}-\gamma y, \bar{x}+\gamma y)|_{\lambda} \geq c^{\prime} y^{2 \lambda+1} .
$$

Finally, inserting this into (4.6) we get

$$
\int_{E}\left(S_{\beta, \kappa} u\right)^{2}(x)|x|^{2 \lambda} d x \geq c^{\prime} \iint_{\Omega^{E_{E}(\alpha, h)}} y\left(\Delta_{\lambda} u^{2}\right)(t, y)|t|^{2 \lambda} d t d y .
$$

The proof of the lemma is completed. 
Lemma 4.4. Let $E$ be a compact subset of $\partial \mathbb{R}_{+}^{2}=\mathbb{R}$, symmetric about $y$-axis, and for $\alpha, h>$ $0, \Omega=\Omega^{E}(\alpha, h)$. Then there exists a family of $y$-symmetric regions $\left\{\Omega_{\epsilon}\right\}_{\epsilon \in(0, h / 3)}$, with the following properties:

(i) $\overline{\Omega_{\epsilon}} \subset \Omega$, and $\Omega_{\epsilon_{1}} \subset \Omega_{\epsilon_{2}}$ if $\epsilon_{2}<\epsilon_{1}$;

(ii) $\Omega_{\epsilon} \rightarrow \Omega$ as $\epsilon \rightarrow 0+$ (i.e., $\cup \Omega_{\epsilon}=\Omega$ );

(iii) The boundary $\partial \Omega_{\epsilon}$ is the union of two parts, $\partial \Omega_{\epsilon}=\mathcal{C}_{\epsilon}^{1} \cup \mathcal{C}_{\epsilon}^{2}$, so that $\mathcal{C}_{\epsilon}^{2}$ is a portion of the horizontal line with $y=h-\epsilon$; and

(iv) $\mathcal{C}_{\epsilon}^{1}$ is a portion of the plane curve $y=\alpha^{-1} \delta_{\epsilon}(x)$ where $\delta_{\epsilon} \in C^{\infty}(\mathbb{R})$, and $\left|\delta_{\epsilon}^{\prime}(x)\right| \leq 1$.

The proof of the lemma follows from that of [44, pp. 206, Lemma 2.2.1], and we only need to check the symmetry of $\Omega_{\epsilon}$. As in [44, pp. 206-207], set $\delta(x)=\operatorname{dist}(x, E)$. Then $\delta$ is a Lipschitz function, and also even, since $E$ is symmetric about $y$-axis. We choose a nonnegative and even $\varphi \in C^{\infty}(\mathbb{R})$, satisfying $\operatorname{supp} \varphi \subset[-1,1]$ and $\int_{\mathbb{R}} \phi(x) d x=1$, and for $\epsilon \in$ $(0, h / 3)$, define $\delta_{\epsilon}(x)=\left(\delta * \varphi_{\alpha \epsilon}\right)(x)+2 \alpha \epsilon$ and $\Omega_{\epsilon}=\left\{(x, y): \delta_{\epsilon}(x)<\alpha y, 0<y<h-\epsilon\right\}$. Obviously $\delta_{\epsilon}$ is even and so $\Omega_{\epsilon}$ is symmetric about $y$-axis. Since $\delta(x)<\delta_{\epsilon}(x), \delta_{\epsilon_{2}}(x)<$ $\delta_{\epsilon_{1}}(x)$ for $\epsilon_{2}<\epsilon_{1}$, and $\delta_{\epsilon}(x)$ tends to $\delta(x)$ as $\epsilon \rightarrow 0+$ uniformly, these $\Omega_{\epsilon}$ 's satisfy the requirements (i)-(iv).

Lemma 4.5 (cf. $[27,28]$ ). Let $\beta, \kappa>0$ be given and $u$ a $\lambda$-harmonic function in $\Gamma_{\beta}^{\kappa}\left(x_{0}\right) \cup$ $\Gamma_{\beta}^{\kappa}\left(-x_{0}\right)$. Then for fixed $\alpha \in(0, \beta)$ and $h \in(0, \kappa)$, there exists a constant $c=c(\alpha, \beta, h, \kappa)>0$, so that

(i) if $|u| \leq 1$ in $\Gamma_{\beta}^{\kappa}\left(x_{0}\right) \cup \Gamma_{\beta}^{\kappa}\left(-x_{0}\right)$, then $y|\nabla u| \leq \operatorname{cin} \Gamma_{\alpha}^{h}\left(x_{0}\right)$;

(ii) if $\left(S_{\beta, \kappa} u\right)\left(x_{0}\right) \leq 1$ and $\left(S_{\beta, \kappa} u\right)\left(-x_{0}\right) \leq 1$, then $y|\nabla u| \leq \operatorname{cin} \Gamma_{\alpha}^{h}\left(x_{0}\right)$.

Now we turn to the proofs of Theorems 4.1 and 4.2.

Proof of Theorem 4.1. For given $\alpha, h>0$, we fix $\beta>\alpha$ and $\kappa>h$. We may assume that $E$ is bounded, without loss of generality. By Lemma 3.1, for each $j \in \mathbb{N}$, there exists a compact set $E_{j} \subset E$, such that $\left|E \backslash E_{j}\right|_{0}<1 / j$, and there is a constant $c_{\beta, \kappa, j}>0$, so that $|u(x, y)| \leq c_{\beta, \kappa, j},(x, y) \in \Omega^{E_{j}}(\beta, \kappa)$. If we put $E_{0}=\bigcup_{j=1}^{\infty} E_{j}$, then $\left|E \backslash E_{0}\right|_{0}=0$. Since $E$ is symmetric about $y$-axis, we may choose each $E_{j}$ preserving this property. Thus, the proof of the theorem would be completed once we prove that for a compact and $y$-symmetric set $E \subset \partial \mathbb{R}_{+}^{2}=\mathbb{R}$,

$$
\int_{E}\left(S_{\alpha, h} u\right)^{2}(x)|x|^{2 \lambda} d x<\infty
$$

under the condition

$$
|u(x, y)| \leq 1 \quad \text { for }(x, y) \in \Omega^{E}(\beta, \kappa),
$$

and by Lemma 4.2 , it suffices to show

$$
\iint_{\Omega^{E}(\alpha, h)} y\left(\Delta_{\lambda} u^{2}\right)(t, y)|t|^{2 \lambda} d t d y<\infty .
$$


Further, for $\Omega=\Omega^{E}(\alpha, h)$, there exists a family of $y$-symmetric regions $\left\{\Omega_{\epsilon}\right\}_{\epsilon>0}$ satisfying (i)-(iv) in Lemma 4.4 , and hence, we only need to prove that, there exists some constant $c>0$ independent of $\epsilon \in(0, h / 3)$, so that

$$
\iint_{\Omega_{\epsilon}} y\left(\Delta_{\lambda} u^{2}\right)(t, y)|t|^{2 \lambda} d t d y<c .
$$

Taking integration over $\Omega_{\epsilon}$ instead of $\Omega$ is for legitimate use of Green's formula. In fact, for $U, V \in C^{2}\left(\overline{\Omega_{\epsilon}}\right)$, by Proposition 2.5 we have

$$
\iint_{\Omega_{\epsilon}}\left(V \Delta_{\lambda} U-U \Delta_{\lambda} V\right)|x|^{2 \lambda} d x d y=\int_{\partial \Omega_{\epsilon}}|x|^{2 \lambda}\left(V \frac{\partial U}{\partial \mathbf{n}}-U \frac{\partial V}{\partial \mathbf{n}}\right) d \ell
$$

If $U=u^{2}$ and $V=y$, then

$$
\iint_{\Omega_{\epsilon}} y\left(\Delta_{\lambda} u^{2}\right)(x, y)|x|^{2 \lambda} d x d y=\int_{\partial \Omega_{\epsilon}}|x|^{2 \lambda}\left(y \frac{\partial u^{2}}{\partial \mathbf{n}}-u^{2} \frac{\partial y}{\partial \mathbf{n}}\right) d \ell .
$$

Since $\partial \Omega_{\epsilon} \subset \Omega^{E}(\beta, \kappa)$, it follows from Lemma 4.5(i) and (4.11) that

$$
\left|y \frac{\partial u^{2}}{\partial \mathbf{n}}\right| \leq 2 y|u||\nabla u| \leq c \quad \text { on } \partial \Omega_{\epsilon}
$$

and since $|\partial y / \partial \mathbf{n}| \leq 1$, we have $\left|u^{2} \partial y / \partial \mathbf{n}\right| \leq 1$ on $\partial \Omega_{\epsilon}$. Applying these estimates to the right hand side of (4.13) and on account of boundedness of $\Omega=\Omega^{E}(\alpha, h)$, we get

$$
\iint_{\Omega_{\epsilon}} y\left(\Delta_{\lambda} u^{2}\right)(x, y)|x|^{2 \lambda} d x d y \leq c \int_{\partial \Omega_{\epsilon}}|x|^{2 \lambda} d \ell \leq c^{\prime} \int_{\partial \Omega_{\epsilon}} d \ell .
$$

Since $E$ is bounded, it follows from [44, pp. 209] that the length of $\partial \Omega_{\epsilon}$ is bounded by a constant independent of $\epsilon$. This proves (4.12) and completes the proof of Theorem 4.1.

Proof of Theorem 4.2. Again, we assume that $E$ is bounded without loss of generality. By the assumption, $E=\cup E_{j}$, where $E_{j}=\left\{x \in E:\left(S_{j^{-1}, j^{-1}} u\right)( \pm x) \leq j\right\}$ which is symmetric about $y$-axis; and for each $j$, by Lemma 4.3 , there exists a sequence of compact and $y$ symmetric subsets $E_{j, k}(k=1, \cdots)$ of $E_{j}$ such that $\left|E_{j} \backslash E_{j, k}\right|_{\lambda}<1 / k$, and for fixed $\alpha \in$ $(0,1 / j)$ and $h \in(0,1 / j)$, there exists some $c=c(\alpha, h, j, k)>0$, such that

$$
\iint_{\Omega^{E_{j, k}(\alpha, h)}} y\left(\Delta_{\lambda} u^{2}\right)(t, y)|t|^{2 \lambda} d t d y \leq c \int_{E_{j}}\left(S_{j^{-1}, j^{-1}} u\right)^{2}(x)|x|^{2 \lambda} d x
$$

For $\left(S_{j^{-1}, j^{-1}} u\right)(x) \leq j\left(x \in E_{j}\right)$ and $E_{j}$ is a bounded set, the left hand side above is finite; and further, by Lemma 4.5 there exists some $c_{1}=c_{1}(\alpha, h, j, k)>0$ such that $y|\nabla u(x, y)| \leq$ $c_{1}$ for $(x, y) \in \Omega^{E_{j}}(\alpha, h)$. Since $\left|E_{j} \backslash \bigcup_{k=1}^{\infty} E_{j, k}\right|_{\lambda}=0$, the proof of the theorem would be 
completed once we prove that, for a compact and $y$-symmetric set $E \subset \partial \mathbb{R}_{+}^{2}=\mathbb{R}, u$ is non-tangentially bounded at $(x, 0)$ for almost every $x \in E$ under the conditions

$$
\iint_{\Omega^{E}(\alpha, h)} y\left(\Delta_{\lambda} u^{2}\right)(x, y)|x|^{2 \lambda} d x d y \leq 1,
$$

for some $\alpha, h>0$, and

$$
y|\nabla u(x, y)| \leq 1 \quad \text { for }(x, y) \in \Omega^{E}(\alpha, h) .
$$

We fix $\alpha_{1} \in(0, \alpha)$ and $h_{1} \in(0, h)$, and work with $\Omega_{\epsilon}\left(\epsilon \in\left(0, h_{1} / 3\right)\right)$ given by Lemma 4.4 associated with $\Omega=\Omega^{E}\left(\alpha_{1}, h_{1}\right)$. By Green's formula (4.13), (4.15) implies

$$
\int_{\partial \Omega_{\epsilon}}|x|^{2 \lambda}\left(y \frac{\partial u^{2}}{\partial \mathbf{n}}-u^{2} \frac{\partial y}{\partial \mathbf{n}}\right) d \ell \leq 1 .
$$

From Lemma 4.4, the boundary $\partial \Omega_{\epsilon}$ of $\Omega_{\epsilon}$ consists of two parts, $\partial \Omega_{\epsilon}=\mathcal{C}_{\epsilon}^{1} \cup \mathcal{C}_{\epsilon}^{2}$, where $\mathcal{C}_{\epsilon}^{1}$ is a portion of the smooth plane curve $y=\alpha_{1}^{-1} \delta_{\epsilon}(x)$ with $\left|\delta_{\epsilon}^{\prime}(x)\right| \leq 1$, and $\mathcal{C}_{\epsilon}^{2}$ is a portion of the horizontal line with $y=h_{1}-\epsilon$.

We extract the term $\int_{\mathcal{C}_{\epsilon}^{1}} u^{2} \frac{\partial y}{\partial \mathbf{n}}|x|^{2 \lambda} d \ell$ from (4.17), so that

$$
-\int_{\mathcal{E}_{\epsilon}^{1}} u^{2} \frac{\partial y}{\partial \mathbf{n}}|x|^{2 \lambda} d \ell \leq \int_{\mathcal{C}_{\epsilon}^{2}} u^{2} \frac{\partial y}{\partial \mathbf{n}}|x|^{2 \lambda} d \ell-\int_{\partial \Omega_{\epsilon}} y \frac{\partial u^{2}}{\partial \mathbf{n}}|x|^{2 \lambda} d \ell+1 .
$$

Since $E$ is bounded and $2 h_{1} / 3 \leq y \leq h_{1}$ for $(x, y) \in \mathcal{C}_{\epsilon}^{2}$, the first term on the right hand side above is bounded by a constant independent of $\epsilon$, and so is the contribution coming from $(x, y) \in \mathfrak{C}_{\epsilon}^{2}$ in the second term. Thus we get

$$
-\int_{\mathcal{C}_{\epsilon}^{1}} u^{2} \frac{\partial y}{\partial \mathbf{n}}|x|^{2 \lambda} d \ell \leq-\int_{\mathcal{C}_{\epsilon}^{1}} y \frac{\partial u^{2}}{\partial \mathbf{n}}|x|^{2 \lambda} d \ell+c .
$$

Since the curve $\mathfrak{C}_{\epsilon}^{1}$ is determined by the equation $\delta_{\epsilon}(x)-\alpha_{1} y=0$ and so the direction $\mathbf{n}$ is given by $\left(\delta_{\varepsilon}^{\prime}(x),-\alpha_{1}\right) / \sqrt{\delta_{\varepsilon}^{\prime}(x)^{2}+\alpha_{1}^{2}}$, we have

$$
\frac{\partial y}{\partial \mathbf{n}}=-\frac{\alpha_{1}}{\sqrt{\delta_{\varepsilon}^{\prime}(x)^{2}+\alpha_{1}^{2}}} \leq-\frac{\alpha_{1}}{\sqrt{1+\alpha_{1}^{2}}} ;
$$

and since $\Omega_{\epsilon} \subset \Omega^{E}\left(a_{1}, h_{1}\right)$ is at a positive distance from $x$-axis, (4.16) implies that

$$
y\left|\frac{\partial u^{2}}{\partial \mathbf{n}}\right|=2 y|u|\left|\frac{\partial u}{\partial \mathbf{n}}\right| \leq 2 y|u||\nabla u| \leq 2|u| \quad \text { on } \quad \mathcal{C}_{\epsilon}^{1} .
$$

Applying these estimates to (4.18) gives

$$
\frac{\alpha_{1}}{\sqrt{1+\alpha_{1}^{2}}} \int_{\mathcal{C}_{\epsilon}^{1}} u^{2}|x|^{2 \lambda} d \ell \leq 2 \int_{\mathcal{C}_{\epsilon}^{1}}|u||x|^{2 \lambda} d \ell+c \leq c^{\prime}\left(\int_{\mathcal{C}_{\epsilon}^{1}} u^{2}|x|^{2 \lambda} d \ell\right)^{1 / 2}+c,
$$


where the last inequality is due to the fact that $\int_{\mathcal{C}_{\epsilon}^{1}}|x|^{2 \lambda} d \ell$ is bounded by a constant independent of $\epsilon$ as in (4.14). This certainly proves that

$$
\int_{\mathcal{C}_{\epsilon}^{1}} u^{2}(x, y)|x|^{2 \lambda} d \ell \leq c,
$$

where $c>0$ is independent of $\epsilon$.

If we define $f_{\epsilon}(x)=\left|u\left(x, \alpha_{1}^{-1} \delta_{\epsilon}(x)\right)\right|$ for $x$ satisfying $(x, y) \in \mathcal{C}_{\epsilon}^{1}$ with some $y>0$, and $f_{\epsilon}(x)=0$ otherwise, then from (4.19), one has, for $\epsilon \in\left(0, h_{1} / 3\right)$,

$$
\int_{\mathbb{R}}\left|f_{\epsilon}(x)\right|^{2}|x|^{2 \lambda} d x \leq \int_{\mathcal{C}_{\epsilon}^{1}} u^{2}(x, y)|x|^{2 \lambda} d \ell \leq c .
$$

Now for $(x, y) \in \mathcal{C}_{\epsilon}^{1} \subset \Omega^{E}\left(\alpha_{1}, h_{1}\right)$ with $0<y<h_{1} / 2$, one can choose a constant $c \in(0,1)$ independent of $(x, y)$, so that the disc $D((x, y) ; c y) \subset \Omega^{E}(\alpha, h)$. It follows that, for $(t, z) \in D((x, y) ; c y),|u(x, y)-u(t, z)| \leq c y$ sup $|\nabla u|$, where the supremum is taken over the line segment joining $(x, y)$ and $(t, z)$, and by (4.16), $|u(x, y)| \leq|u(t, z)|+c$. We then take the curve integration over $\mathcal{C}_{\epsilon}^{1} \cap D((x, y) ; c y)$, and in view of the inequality, for some fixed $c_{1} \in(0, c)$,

$$
\int_{\mathcal{C}_{\epsilon}^{1} \cap D((x, y) ; c y)}|t|^{2 \lambda} d \ell \geq \int_{x-c_{1} y}^{x+c_{1} y}|t|^{2 \lambda} d t \asymp y(|x|+y)^{2 \lambda},
$$

we get

$$
|u(x, y)| \leq \frac{c^{\prime}}{y(|x|+y)^{2 \lambda}} \int_{\mathcal{E}_{\epsilon}^{1} \cap D((x, y) ; c y)}|u(t, z)||t|^{2 \lambda} d \ell+c .
$$

Thus by means of (3.3), for $(x, y) \in \mathcal{C}_{\epsilon}^{1}$ with $0<y<h_{1} / 2$,

$$
\begin{aligned}
|u(x, y)| & \leq c^{\prime} \int_{\mathcal{C}_{\epsilon}^{1} \cap D((x, y) ; c y)}|u(t, z)|\left(\tau_{x} P_{y}\right)(-t)|t|^{2 \lambda} d \ell+c \\
& \leq c^{\prime \prime} v_{\epsilon}(x, y)+c
\end{aligned}
$$

where

$$
v_{\epsilon}(x, y)=c_{\lambda} \int_{\mathbb{R}} f_{\epsilon}(t)\left(\tau_{x} P_{y}\right)(-t)|t|^{2 \lambda} d t
$$

is the $\lambda$-Poisson integral of $f_{\epsilon}$. Further, since $u$ has a bound on $\left\{(x, y) \in \partial \Omega_{\epsilon}: h_{1} / 2 \leq\right.$ $\left.y \leq h_{1}-\epsilon\right\}$ independent of $\epsilon$, we could choose the constant $c$ suitably large, so that (4.21) is true for all $(x, y) \in \partial \Omega_{\epsilon}$.

Considering the function

$$
U(x, y)=|u(x, y)|-c^{\prime \prime} v_{\epsilon}(x, y)-c,
$$

by Lemma 3.2 we have

$$
\Delta_{\lambda} U(x, y)=\Delta_{\lambda}|u(x, y)| \geq 0
$$


in the region where $U(x, y)>0$, which implies $|u(x, y)|>0$. Since $\left.U\right|_{\partial \Omega_{\epsilon}} \leq 0$ from (4.21), by the maximum principle (Proposition 2.6) we assert that (4.21) holds on the whole $\Omega_{\epsilon}$.

Finally, since, from (4.20), $\left\{f_{\epsilon}: \epsilon \in\left(0, h_{1} / 3\right)\right\}$ is a bounded set in $L_{\lambda}^{2}(\mathbb{R})$, there exists a sequence $\left\{f_{\epsilon_{k}}\right\}_{k=1}^{\infty}$, so that $f_{\varepsilon_{k}}$ converges weakly to a function $f \in L_{\lambda}^{2}(\mathbb{R})$ as $k \rightarrow \infty$; and in particular, if $v(x, y)$ denotes the $\lambda$-Poisson integral of $f$, then $v_{\epsilon_{k}}(x, y)$ converges pointwise to $v(x, y)$ for $(x, y) \in \mathbb{R}_{+}^{2}$. Thus, since by Lemma $4.4, \Omega_{\epsilon_{j}}$ approaches increasingly to $\Omega^{E}\left(a_{1}, h_{1}\right)$, we conclude from (4.21) that

$$
|u(x, y)| \leq c^{\prime \prime} v(x, y)+c, \quad(x, y) \in \Omega^{E}\left(a_{1}, h_{1}\right) .
$$

By Proposition 2.4, $v(x, y)$ is non-tangentially bounded at $(x, 0)$ for almost every $x \in \mathbb{R}$, and hence, the same is true for $u(x, y)$ and for almost every $x \in E$. The proof of Theorem 4.2 is completed.

\section{Acknowledgements}

The work was supported by the National Natural Science Foundation of China (No. 11371258).

\section{References}

[1] L. Atanasi and M. A. Picardello, The Lusin area function and local admissible convergence of harmonic functions on homogeneous trees, Trans. Amer. Math. Soc., 360 (2008), 3327-3343.

[2] M. Brelot and J. L. Doob, Limites angulaires et limites fines, Annales de l'Institut Fourier (Grenoble), 13 (1963), 395-415.

[3] J. Brossard, Comportement non-tangentiel et comportement brownien des fonctions harmoniques dans un demi-espace. Démonstration probabiliste d'un théorème de Calderon et Stein, Séminaire de Probabilités, XII (Univ. Strasbourg, Strasbourg, 1976/1977), 378-397, Lecture Notes in Mathematics 649, Springer, Berlin, 1978.

[4] J. Brossard, Densité de l'intégrale d'aire dans $\mathbb{R}_{+}^{n+1}$ et limites non tangentielles, Invent. Math., 93 (1988), 297-308.

[5] A. P. Calderón, On the behaviour of harmonic functions at the boundary, Trans. Amer. Math. Soc., 68 (1950), 47-54.

[6] A. P. Calderón, On a theorem of Marcinkiewicz and Zygmund, Trans. Amer. Math. Soc., 68 (1950), 55-61.

[7] L. Carleson, On the existence of boundary values for harmonic functions of several variables, Ark. Mat., 4 (1962), 393-399.

[8] M. F. E. de Jeu, The Dunkl transform, Invent. Math., 113 (1993), 147-162.

[9] C. F. Dunkl, Reflection groups and orthogonal polynomials on the sphere, Math. Z., 197 (1988), 33-60.

[10] C. F. Dunkl, Differential-difference operators associated to reflection groups, Trans. Amer. Math. Soc., 311 (1989), 167-183.

[11] C. F. Dunkl, Integral kernels with reflection group invariance, Canad. J. Math., 43 (1991), $1213-1227$. 
[12] C. F. Dunkl, Hankel transforms associated to finite reflection groups, in Proceedings of the special session on hypergeometric functions on domains of positivity, Jack polynomials and applications (Tampa, 1991), Contemp. Math., 138 (1992), 123-138.

[13] C. F. Dunkl, Reflection groups in analysis and applications, Japan J. Math., 3(2) (2008), 215246.

[14] R. F. Gundy, The density of the area integral, Conference on Harmonic Analysis in Honor of Antoni Zygmund, Vols. I, II (Chicago, Ill., 1981), 138-149, Wadsworth Mathematics Series, Belmont, Wadsworth, CA, 1983.

[15] R. A. Hunt and R. L. Wheeden, On the boundary values of harmonic functions, Trans. Amer. Math. Soc., 132 (1968), 307-322.

[16] R. A. Hunt and R. L. Wheeden, Positive harmonic functions on Lipschitz domains, Trans. Amer. Math. Soc., 147 (1970), 507-527.

[17] D. Jerison and C. Kenig, Boundary behaviour of harmonic functions in nontangentially accessible domains, Adv. Math., 46 (1982), 80-147.

[18] A. Koráányi, Harmonic functions on Hermitian hyperbolic space, Trans. Amer. Math. Soc., 135 (1969), 507-516.

[19] A. Korányi, Boundary behavior of Poisson integrals on symmetric spaces, Trans. Amer. Math. Soc., 140 (1969), 393-409.

[20] A. Korányi and R. B. Putz, Local Fatou theorem and area theorem for symmetric spaces of rank one, Trans. Amer. Math. Soc., 224 (1976), 157-168.

[21] S. G. Krantz, Invariant metrics and the boundary behavior of holomorphic functions on domains in $\mathbb{C}^{n}$, J. Geometric Anal., 1 (1991), 71-98.

[22] S. G. Krantz, The boundary behavior of holomorphic functions: global and local results, Asian J. Math., 11 (2007), 179-200.

[23] L. Lapointe and L. Vinet, Exact operator solution of the Calogero-Sutherland model, Commun. Math. Phys., 178 (1996), 425-452.

[24] L. Lempert, Boundary behavior of meromorphic functions of several complex variables, Acta Math., 144 (1980), 1-26.

[25] Zh.-K. Li and J.-Q. Liao, Harmonic analysis associated with the one-dimensional Dunkl transform, Constr. Approx., 37 (2013), 233-281.

[26] Zh.-K. Li and J.-Q. Liao, Hardy spaces for Dunkl-Gegenbauer expansions, J. Funct. Anal., 265 (2013), 687-742.

[27] J.-Q. Liao, Harmonic Analysis in the One-Parameter Dunkl Setting, Ph.D dissertation, Capital Normal University, Beijing, 2013.

[28] J.-Q. Liao and Zh.-K. Li, Area integrals associated with the rank-one Dunkl operator, preprint.

[29] J.-Q. Liao, X.-L. Zhang and Zh.-K. Li, On Littlewood-Paley functions associated with the Dunkl operator, Bull. Austr. Math. Soc., 96 (2017), 127-138.

[30] J. Marcinkiewicz and A. Zygmund, A theorem of Lusin, Duke Math. J., 4 (1938), 473-485.

[31] B. Muckenhoupt and E. M. Stein, Classical expansions and their relation to conjugate harmonic functions, Trans. Amer. Math. Soc., 118 (1965), 17-92.

[32] F. Mouton, Comportement asymptotique des fonctions harmoniques en courbure négative, Commentarii Mathematici Helvetici, 70 (1995), 475-505.

[33] F. Mouton, Comportement asymptotique des fonctions harmoniques sur les arbres, Séminaire de Probabilités, XXXIV, 353-373, Lecture Notes in Mathematics 1729, Springer, Berlin, 2000.

[34] F. Mouton, Local Fatou theorem and the density of energy on manifolds of negative curva- 
ture, Revista Mate. Iberoam., 23 (2007), 1-16.

[35] C. Petit, Harmonic functions on hyperbolic graphs, Proc. Amer. Math. Soc., 140 (2012), 235248.

[36] C. Petit, Boundary behavior of harmonic functions on Gromov hyperbolic manifolds, Int. Math. Res. Not., 1 (2015), 212-239.

[37] M. A. Picardello, Local admissible convergence of harmonic functions on non-homogeneous trees, Colloquium Math., 118 (2010), 419-944.

[38] I. Privalov, Sur une généralisation du théorème de Fatou, Rec. Math., (Mat. Sbornik) 31 (1923), 232-235.

[39] R. B. Putz, A generalized area theorem for harmonic functions on Hermitian hyperbolic space, Trans. Amer. Math. Soc., 168 (1972), 243-258.

[40] M. Rösler, Bessel-type signed hypergroups on $\mathbb{R}$, in "Probability measures on groups and related structures XI", Edited by H. Heyer and A. Mukherjea, pp. 292-304, World Scientific, Singapore, 1995.

[41] M. Rösler, Dunkl operators: theory and applications, in Orthogonal Polynomials and Special Function, Lecture Notes in Math., 1817, Edited by E. Koelink and W. Van Assche, SpringerVerlag, Berlin, 2003, pp. 93-135.

[42] D. C. Spencer, A function theoretic identity, Amer. J. Math., 65 (1943), 147-160.

[43] E. M. Stein, On the theory of harmonic functions of several variables II: Behavior near the boundary, Acta Math., 106 (1961), 137-174.

[44] E. M. Stein, Singular Integrals and Differentiability Properties of Functions, Princeton University Press, 1970.

[45] S. Thangavelu and Y. Xu, Convolution operator and maximal function for Dunkl transform, J. Anal. Math., 97 (2005), 25-55.

[46] K. O. Widman, On the boundary values of harmonic functions in $\mathbb{R}^{3}$, Ark. Mat., 5 (1964), 221-230. 